\title{
Anthropometric and Physical Fitness Profiles of World-Class Male Padel Players
}

\author{
Cristóbal Sánchez-Muñoz ${ }^{1}$, José Joaquín Muros ${ }^{2, *} \mathbb{1}$, Jerónimo Cañas ${ }^{3}$, Javier Courel-Ibáñez ${ }^{4}$, \\ Bernardino Javier Sánchez-Alcaraz ${ }^{4}$ and Mikel Zabala ${ }^{3}$ \\ 1 Department of Physical Education and Sport, University of Granada, 52071 Melilla, Spain; csm@ugr.es \\ 2 Department of Didactics of Musical, Plastic and Corporal Expression, University of Granada, \\ 18071 Granada, Spain \\ 3 Department of Physical Education and Sport, University of Granada, 18071 Granada, Spain; \\ jerocanias@yahoo.com (J.C.); mikelz@ugr.es (M.Z.) \\ 4 Department of Physical Education and Sport, University of Murcia, 30720 Murcia, Spain; \\ javier.courel.ibanez@gmail.com (J.C.-I.); bjavier.sanchez@um.es (B.J.S.-A.) \\ * Correspondence: jjmuros@ugr.es
}

Received: 13 December 2019; Accepted: 10 January 2020; Published: 13 January 2020

\begin{abstract}
The aims of this study were to describe and compare the anthropometric and physical fitness attributes of male padel players according to their competitive level, and to establish a functional anthropometric and physical profile. A total of 60 males participated in the present study. Athletes were grouped according to competition level, forming an elite group $(n=25)$ and a subelite group $(n=35)$. Anthropometric variables, hand grip and lumbar isometric strength, flexibility, and lower-body muscular strength were measured. Elite padel players were significantly older and showed significantly lower values for the thigh and calf skinfolds, the sum of six and eight skinfolds, and the sum of lower-limb skinfolds than the subelite group. Elite padel players also had significantly lower values than subelite players for body fat percentage and thigh fat area, whilst showing significantly higher values for lumbar isometric strength. Somatotype of the elite padel players could be defined as endo-mesomorphic. Results suggest that training and talent identification of padel players should focus on their anthropometric characteristics and physical fitness, with these being different between elite and subelite athletes. Normative data could help coaches throughout the talent identification process and in the design of training programs when seeking to optimise sports performance.
\end{abstract}

Keywords: body composition; somatotype; anthropometry; physical fitness; padel

\section{Introduction}

Padel is a modern racket sport that was invented in the 1970s. With interest growing in recent years, padel has now become one of the most popular sports in Spain with over four million regular participants and it is rapidly spreading around the world [1]. In padel, two pairs of players confront each other inside an enclosed synthetic glass and metal court $(20 \times 10$ meters). An important peculiarity is the existence of walls and grilles surrounding the court off of which the ball can bounce. This consequently lengthens rallies so the number of actions and strokes per player is higher in comparison with similar racket sports like badminton, tennis, or squash [2]. Previous studies examining its game dynamics and match activity have defined padel as a high-intensity intermittent activity, which combines high-frequency (0.7-1.5 per second (s)) and low-intensity actions during rallies that are of a moderate duration (9-15 s), interspersed by $1020 \mathrm{~s}$ of rest in between, leading to longer breaks of $90 \mathrm{~s}$ [3-7]. Offensive success in padel relies on the use of volleys and smashing actions. Defensive actions are 
focused on sending the opponent to the back of the court though lobs and bouncing the ball off the wall from the baseline [5]. Thus, match activity in padel can be considered as a mix between tennis and squash $[8,9]$.

Aside from game dynamics and technical-tactical demands, performance is also related with physical fitness and anthropometric measures. Particularly in racket sports, body kinematics and anthropometric parameters are associated with greater success $[10,11]$. Furthermore, recent research suggests that physical fitness has little impact on determining padel players' performance at nonprofessional levels $[12,13]$. This may be due to the less demanding competitive requirements at a recreational level. Nonetheless, the increasing intensity of sport competitions at a professional level make strength and conditioning training a priority for success [14]. Although a few studies have examined anthropometric characteristics in amateur padel players $[4,12,15]$, there is no information available to confirm these results in top level players. The determination of normative profiles for professional padel players would assist coaches in the talent identification process and improve the design of specific training in order to maximize performance.

The aims of the present study were (1) to describe and compare the anthropometric and physical fitness attributes of male padel players at different competitive levels, (2) to establish an anthropometric and physical profile chart for professional male padel players, and (3) to compare results of the present study with outcomes obtained in other racket sports.

\section{Materials and Methods}

\subsection{Subjects}

Sixty male padel players ( $27.7 \pm 6.4$ years $)$ volunteered to participate in this study. They were participants at two Open events on the 2012 Padel Pro Tour (PPT) in Marbella and Fuengirola (Spain). PPT is the professional padel circuit, which was created between 2005 and 2013, and is currently called World Padel Tour (WPT). It constitutes the most important professional padel tournament in the world. Participants were classified according to competition level as (1) an elite group ( $n=25)$ who competed in the Main Draw of PPT events (members of the top 28 pairings in the PPT ranking), and (2) a subelite group $(n=35)$ who competed in a Qualifying Round or in a Prequalifying Round for these events (players who were ranked below the top 28 pairings in the PPT ranking). Players were fully informed about the experimental procedures, including the risks and benefits of participation. Written informed consent was obtained from each player prior to data collection. The study was approved by the Ethics Committee of the University of Granada (number 883) and was carried out in compliance with the Declaration of Helsinki.

\subsection{Anthropometric Data}

Anthropometric measurements were performed according to guidelines outlined by the International Society for Advancement of Kinanthropometry (ISAK) [16]. All anthropometric variables were taken by the same experienced evaluator who was a Level 2 ISAK anthropometrist. Technical measurement error was less than $5 \%$ for skinfolds and less than $1 \%$ for all other measurements. Anthropometric variables included stature, body mass, arm span, 8 skinfolds (biceps, triceps, subscapular, suprailiac, supraspinal, abdominal, thigh, and medial calf), 7 girths (flexed and tensed upper arm, right and left forearms, wrist, thigh, and medial calf), 2 breadths (humeral and femoral), and width and length of the hands (dominant and nondominant). Stature was measured to the nearest $0.1 \mathrm{~cm}$ using a stadiometer (GPM, Seritex, Inc., Carlstadt, New Jersey), and body mass was measured to the nearest $0.1 \mathrm{~kg}$ using a portable scale (model 707, Seca Corporation, Columbia, Maryland). Skinfold thickness was obtained using a Holtain skinfold caliper (Holtain Ltd., Crymych, UK) and recorded to the nearest $0.2 \mathrm{~mm}$. Girths were measured using a flexible anthropometric steel tape (Holtain Ltd., Crymych, UK) to the nearest $0.1 \mathrm{~cm}$. Skinfolds were taken 3 times and the median was used in analyses. The sum of 3 skinfolds (triceps, subscapular, and supraspinal), 6 skinfolds (sum of 3 with suprailiac, 
abdominal, and thigh), and 8 skinfolds (sum of 6 with biceps and medial calf) was also calculated. Body mass index (BMI) was calculated as body mass/stature ${ }^{2}$ where body mass was expressed in kilograms $(\mathrm{kg})$ and stature in metres $(\mathrm{m})$. Five different equations [17-21] were used to estimate body density. Body fat percentage (BF\%) was determined using Siri's equation [22]. Muscle mass (MM) was determined in $\mathrm{kg}$ using the methods of Lee et al. [23]. Somatotype characteristics were determined according to the Carter and Heath method [24].

\subsection{Lower-Body Muscular Strength}

Following determination of anthropometric variables, players performed a warm-up consisting of several submaximal jumps. Lower-body muscular strength was assessed using the Infrared Platform Ergo Jump Plus-Bosco System (Byomedic, S.C.P., Barcelona, Spain). Each player completed three maximal countermovement jumps (CMJs) with 3 minutes rest between trials. The best score was recorded. All CMJs were completed keeping the hands on the hips throughout the test. Whilst standing erect, participants were instructed to flex their knees into a squat position (90) and then immediately rebound in a maximal vertical jump. No pause was allowed between the eccentric and concentric phase, and participants landed with both feet in contact with the floor. Measured height was expressed in centimetres and was converted to power $(w)$ using the González-Badillo and Gorostiaga equation [25]:

$$
\text { Power }(w)=\text { weight }(\mathrm{kg}) \times 9.81 \times[\sqrt{2 \times 9.81 \times \text { jump height }(\mathrm{m})}]
$$

\subsection{Handgrip Strength}

A grip strength dynamometer (Takei Kiki Kogyo, Tokyo, Japan) was used to determine handgrip strength in both right and left hands. The dynamometer was adjusted for each participant's hand size. Players maintained a standing position with the shoulder adducted and neutrally rotated and the elbow fully extended. The dynamometer was held freely without support, not touching the subject's trunk. Players were instructed to perform a maximal isometric contraction for five seconds. Each participant completed three trials with each hand, with a $1 \mathrm{~min}$ rest between trials, and highest scores (in $\mathrm{kg}$ ) were recorded.

\subsection{Lumbar Isometric Strength}

A lumbar extension dynamometer (Takei Kiki Kogyo, Tokyo, Japan) was used to determine lumbar isometric strength. Players stood on the platform with knees extended and the trunk flexed to an angle of $150^{\circ}$. Holding the bar with a pronated grip, the participant pulled it slowly, but vigorously, extending the lower back. Players were instructed to perform a maximal isometric contraction for five seconds. The best score of three trials with $1 \mathrm{~min}$ recovery between each was recorded and used in the analyses.

\subsection{Flexibility}

The sit-and-reach test was used to assess lower-body flexibility. A sit-and-reach box (Novel Products, Inc., Rockton, Illinois, USA) with a scale marked on the upper side was placed against a wall. Players removed their shoes and sat on the floor with their legs fully extended and feet against the box. Placing one hand on top of the other and keeping their legs straight, players reached forward as far as possible whilst sliding their fingers along the measurement scale on top of the box. Players were asked to hold the final position for three seconds, and measurements were recorded to the nearest centimetre. After a familiarization practice, each player performed three trials with the best score being recorded for analysis. 


\subsection{Statistical Analyses}

Variables are described as mean, standard deviation, and range. The standardising of variables was carried out using the Shapiro-Wilk test. Differences in physical and anthropometric variables between the elite group and the subelite group of padel players were analysed using an independent $t$-test. Statistical significance was stablished at $5 \%$. A profile chart with norms determined according to percentiles (values of 5, 10, 25, 50, 75, 90, and 95) was constructed for the elite group of male padel players. All statistical analyses were performed using the Statistical Package for Social Sciences (version 21.0; SPSS, Inc, Chicago, Illinois, USA).

\section{Results}

Demographic characteristics of the sample are presented in Table 1. Comparisons between groups showed that the elite padel players were significantly older ( $31 \pm 5.7 \mathrm{vs.} 25.3 \pm 5.9$ years), had significantly lower values for the thigh $(10.6 \pm 3.8$ vs. $14.1 \pm 5.7 \mathrm{~mm})$ and calf $(6.7 \pm 2.3$ vs. $9.3 \pm 4.4$ $\mathrm{mm})$ skinfolds, the sum of six and eight skinfolds $(84.5 \pm 30.7$ vs. $102.7 \pm 38.5 \mathrm{~mm}$ and $95.3 \pm 33.3$ vs. $116 \pm 43.2 \mathrm{~mm}$, respectively), and the sum of lower-limb skinfolds ( $17.3 \pm 5.4$ vs. $23.5 \pm 9.9 \mathrm{~mm})$ than the subelite group. In the same way, elite padel players presented lower values for $\%$ body fat evaluated by Sloan [19], Wilmore and Behnke [20], and Whiters et al. [21] equations $(9.7 \pm 3.3$ vs. $12.5 \pm 4.7 \%, 14.2 \pm 3.6$ vs. $16.3 \pm 4.2 \%$, and $11.9 \pm 4.1$ vs. $14.6 \pm 5.4 \%$, respectively), and thigh fat area $\left(27.5 \pm 9.4\right.$ vs. $\left.36.6 \pm 15.4 \mathrm{~cm}^{2}\right)$. Furthermore, elite players reported engaging in padel for longer ( $15.0 \pm 6.1$ vs. $8.6 \pm 3.9$ years) and showed significantly higher values for lumbar isometric strength $(147.1 \pm 43.5$ vs. $126.9 \pm 30.4 \mathrm{~kg})$ than the subelite group. The mean somatotype for elite padel players was: 3.7-5.7-2.0. This demonstrates that these players were predominantly mesomorphic, being characterised as endo-mesomorphic according to Carter and Heath's [24] classification. Figure 1 shows the somatoplots for all individual players.

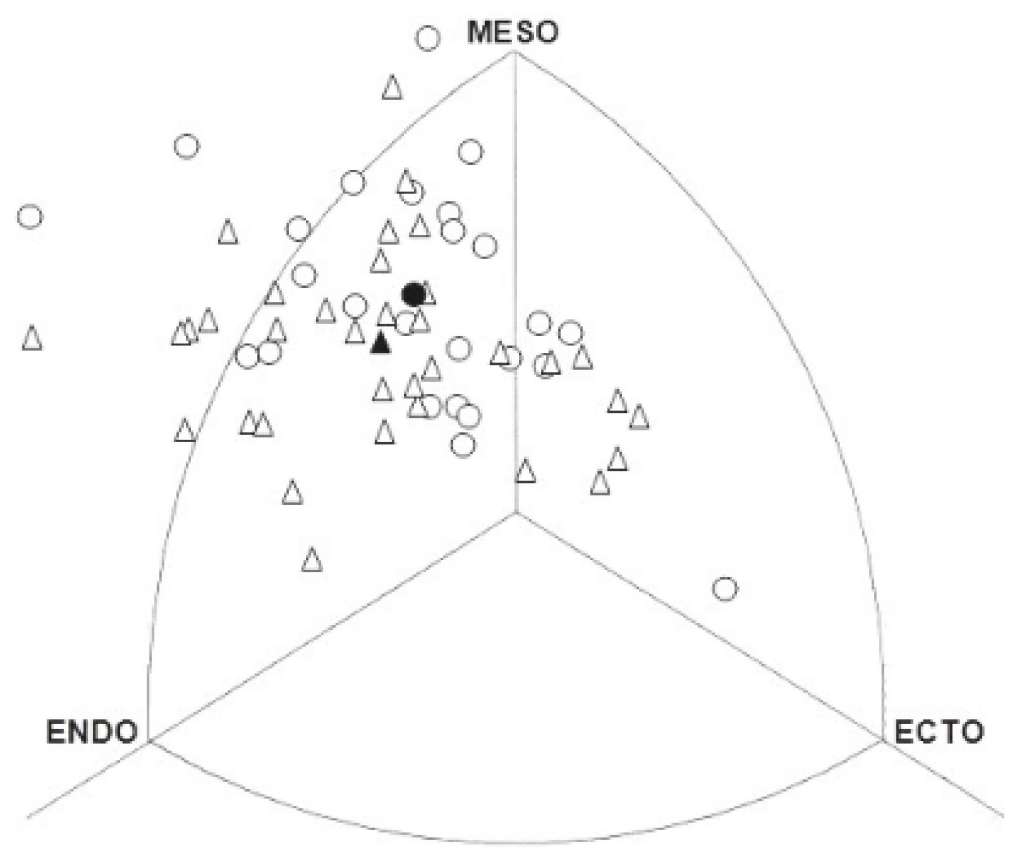

Figure 1. Somatotype distribution of elite $(\bullet:$ Mean somatotype $=3.7-5.7-2.0 ; \bigcirc:$ Individual somatotype $)$ and subelite $(\boldsymbol{\Lambda}$ : Mean somatotype $=4.3-5.3-2.1 ; \Delta$ : Individual somatotype $)$ padel players. MESO: mesomorphy; ECTO: ectomorphy; ENDO: endomorphy. 
Table 1. Anthropometric and physical characteristics of the total sample, and elite and subelite padel players.

\begin{tabular}{|c|c|c|c|c|c|c|c|c|}
\hline \multirow{2}{*}{ Variables } & \multicolumn{2}{|c|}{ Total Padel Players $(n=60)$} & \multicolumn{2}{|c|}{ Elite $(n=25)$} & \multicolumn{2}{|c|}{ Subelite $(n=35)$} & \multirow{2}{*}{$p$-Value } & \multirow{2}{*}{ Cohen's $d$} \\
\hline & Mean \pm SD & Range & Mean \pm SD & Range & Mean \pm SD & Range & & \\
\hline Age (yr) & $27.7 \pm 6.4$ & $15.2-40.2$ & $31.1 \pm 5.7$ & $18.1-38.3$ & $25.3 \pm 5.9$ & $15.2-40.2$ & 0.000 ** & 0.505 \\
\hline Total years playing padel $(\mathrm{yr})$ & $12.5 \pm 6.1$ & $4.0-22.0$ & $15.0 \pm 6.1$ & $8.0-22.0$ & $8.6 \pm 3.9$ & $4.0-16.0$ & $0.010^{*}$ & 1.250 \\
\hline Training (hours/week) & $9.2 \pm 7.3$ & $2.0-25.0$ & $11.4 \pm 7.8$ & $2.0-25.0$ & $5.8 \pm 5.2$ & $2.0-18.0$ & 0.091 & 0.844 \\
\hline Stature $(\mathrm{cm})$ & $178.0 \pm 7.5$ & $163.5-200.2$ & $177.7 \pm 7.3$ & $163.5-196.2$ & $178.3 \pm 7.7$ & $164.1-200.2$ & 0.772 & -0.080 \\
\hline Body weight $(\mathrm{kg})$ & $77.5 \pm 10.0$ & $56.0-101.6$ & $77.2 \pm 9.9$ & $56.0-97.9$ & $77.6 \pm 10.2$ & $56.8-101.6$ & 0.879 & -0.040 \\
\hline $\operatorname{BMI}\left(\mathrm{kg} / \mathrm{m}^{2}\right)$ & $24.4 \pm 2.3$ & $18.5-31.4$ & $24.4 \pm 2.5$ & $18.5-31.4$ & $24.4 \pm 2.3$ & $19.0-28.7$ & 0.933 & 0 \\
\hline Arm span $(\mathrm{cm})$ & $182.3 \pm 8.3$ & $161.5-203.5$ & $182.3 \pm 7.6$ & $161.5-203.5$ & $182.3 \pm 8.9$ & $164.5-201.5$ & 0.990 & 0 \\
\hline Triceps skinfold $(\mathrm{mm})$ & $11.5 \pm 4.9$ & $5.2-26.8$ & $10.5 \pm 4.4$ & $5.4-22.6$ & $12.1 \pm 5.2$ & $5.2-26.8$ & 0.204 & -0.332 \\
\hline Biceps skinfold (mm) & $4.6 \pm 1.9$ & $2.0-11.0$ & $4.1 \pm 1.5$ & $2.4-8.2$ & $4.9 \pm 2.1$ & $2.6-11.2$ & 0.120 & -0.438 \\
\hline Subscapular skinfold (mm) & $10.9 \pm 4.2$ & $6.2-27.0$ & $10.1 \pm 4.1$ & $6.4-27.0$ & $11.5 \pm 4.2$ & $6.2-23.2$ & 0.197 & -0.337 \\
\hline Suprailiac skinfold (mm) & $20.6 \pm 8.7$ & $8.2-39.2$ & $18.3 \pm 7.2$ & $9.4-34.2$ & $22.2 \pm 9.3$ & $8.2-39.2$ & 0.083 & -0.469 \\
\hline Supraspinale skinfold (mm) & $9.9 \pm 5.3$ & $4.0-31.2$ & $8.8 \pm 4.2$ & $4.2-22.2$ & $10.7 \pm 5.9$ & $4.0-31.2$ & 0.168 & -0.371 \\
\hline Abdominal skinfold (mm) & $18.9 \pm 9.4$ & $5.6-37.0$ & $16.8 \pm 8.7$ & $6.2-36.2$ & $20.5 \pm 9.7$ & $5.6-37.0$ & 0.131 & -0.402 \\
\hline Thigh skinfold $(\mathrm{mm})$ & $12.7 \pm 5.3$ & $5.8-31.6$ & $10.6 \pm 3.8$ & $5.8-22.4$ & $14.1 \pm 5.7$ & $6.6-31.6$ & 0.009 * & -0.723 \\
\hline Calf skinfold (mm) & $8.3 \pm 3.9$ & $4.0-22.0$ & $6.7 \pm 2.3$ & $4.2-11.8$ & $9.3 \pm 4.4$ & $4.0-22.0$ & 0.004 * & -0.741 \\
\hline Upper-arm girth $(\mathrm{cm})^{+}$ & $31.5 \pm 2.5$ & $25.8-37.1$ & $31.8 \pm 2.6$ & $26.3-37.1$ & $31.3 \pm 2.4$ & $25.8-36.3$ & 0.434 & 0.200 \\
\hline Upper-arm girth $(\mathrm{cm})^{t+}$ & $33.6 \pm 2.6$ & $28.5-29.7$ & $33.9 \pm 2.6$ & $28.2-38.0$ & $33.4 \pm 2.6$ & $27.8-39.8$ & 0.535 & 0.192 \\
\hline Right forearm girth $(\mathrm{cm})$ & $28.7 \pm 1.8$ & $24.6-33.2$ & $28.8 \pm 2.1$ & $25.8-33.2$ & $28.6 \pm 1.7$ & $24.6-31.5$ & 0.659 & 0.105 \\
\hline Left forearm girth $(\mathrm{cm})$ & $27.5 \pm 1.6$ & $23.3-31.1$ & $27.5 \pm 1.8$ & $23.3-31.1$ & $27.4 \pm 1.4$ & $23.9-29.8$ & 0.804 & 0.062 \\
\hline Wrist girth $(\mathrm{cm})$ & $17.4 \pm 1.0$ & $15.3-20.0$ & $17.5 \pm 1.1$ & $15.6-20.0$ & $17.2 \pm 0.8$ & $15.3-19.0$ & 0.200 & 0.312 \\
\hline $\begin{array}{l}\text { Thigh girth }(\mathrm{cm}) \\
\text { The }\end{array}$ & $53.8 \pm 3.4$ & $46.2-61.7$ & $53.9 \pm 3.2$ & $47.2-61.7$ & $53.7 \pm 3.6$ & $\begin{array}{l}46.2-60.4 \\
46.0\end{array}$ & 0.821 & 0.059 \\
\hline Calf girth (maximum) $(\mathrm{cm})$ & $37.8 \pm 2.1$ & $32.6-42.1$ & $37.8 \pm 2.3$ & $32.6-41.8$ & $37.8 \pm 1.9$ & $33.1-42.1$ & 0.975 & 0 \\
\hline Humerus breadth $(\mathrm{cm})$ & $7.2 \pm 0.4$ & $6.2-8.4$ & $7.3 \pm 0.5$ & $6.2-8.4$ & $7.1 \pm 0.4$ & $6.4-8.0$ & 0.240 & 0.442 \\
\hline Femur breadth $(\mathrm{cm})$ & $10.1 \pm 0.5$ & $8.9-11.3$ & $10.1 \pm 0.5$ & $9.3-11.3$ & $10.0 \pm 0.5$ & $8.9-11.0$ & 0.395 & 0.200 \\
\hline Dominant hand length $(\mathrm{cm})$ & $20.0 \pm 1.1$ & $17.7-22.8$ & $20.0 \pm 1.1$ & $17.7-22.2$ & $20.0 \pm 1.1$ & $18.5-22.8$ & 0.846 & 0 \\
\hline Dominant hand width $(\mathrm{cm})$ & $22.4 \pm 1.7$ & $18.9-26.5$ & $22.3 \pm 1.9$ & $19.5-25.9$ & $22.5 \pm 1.5$ & $18.9-26.5$ & 0.746 & -0.117 \\
\hline Nondominant hand length $(\mathrm{cm})$ & $19.8 \pm 1.2$ & $16.6-23.5$ & $19.8 \pm 1.3$ & $16.6-22.8$ & $19.9 \pm 1.1$ & $18.3-23.5$ & 0.728 & -0.083 \\
\hline Nondominant hand width $(\mathrm{cm})$ & $22.7 \pm 1.6$ & $19.3-26.7$ & $22.6 \pm 1.9$ & $19.7-25.9$ & $22.9 \pm 1.4$ & $19.3-26.7$ & 0.563 & -0.180 \\
\hline Sum of 3 skinfolds (mm) & $42.9 \pm 16.6$ & $19.6-88.0$ & $38.9 \pm 14.6$ & $22.2-83.8$ & $45.8 \pm 17.5$ & $19.6-88.0$ & 0.109 & -0.428 \\
\hline Sum of 6 skinfolds $(\mathrm{mm})$ & $95.1 \pm 36.4$ & $40.0-186.0$ & $84.5 \pm 30.7$ & $49.0-164.6$ & $102.7 \pm 38.5$ & $40.0-186.2$ & 0.047 * & -0.523 \\
\hline Sum of 8 skinfolds (mm) & $107.9 \pm 40.5$ & $49.0-210.0$ & $95.3 \pm 33.3$ & $58.0-184.4$ & $116.9 \pm 43.2$ & $48.6-210.4$ & $0.041 *$ & -0.560 \\
\hline Sum upper-limb skinfolds ( $\mathrm{mm}$ ) & $76.3 \pm 31.6$ & $32.2-150.4$ & $68.5 \pm 27.8$ & $36.6-150.4$ & $81.9 \pm 33.3$ & $32.2-150.2$ & 0.105 & -0.437 \\
\hline Sum lower-limb skinfolds (mm) & $20.9 \pm 8.8$ & $10.2-53.0$ & $17.3 \pm 5.4$ & $10.2-34.2$ & $23.5 \pm 9.9$ & $11.6-53.0$ & 0.003 * & -0.798 \\
\hline \multicolumn{9}{|l|}{$\%$ body fat } \\
\hline Durnin and Womersley [17] & $19.7 \pm 5.3$ & $10.1-31.2$ & $18.4 \pm 4.8$ & $11.7-30.4$ & $20.7 \pm 5.4$ & $10.1-31.2$ & 0.106 & -0.450 \\
\hline Katch and McArdle [18] & $13.4 \pm 5.1$ & $6.3-27.4$ & $12.2 \pm 4.7$ & $7.2-26.7$ & $14.2 \pm 5.3$ & $6.3-27.4$ & 0.139 & -0.399 \\
\hline $\begin{array}{l}\text { Sloan [19] } \\
\text { Slite [10] }\end{array}$ & $\begin{array}{l}10.4 \pm 3.1 \\
11.3 \pm 4.4\end{array}$ & $5.2-24.0$ & $\begin{array}{l}1.2 \pm 4.7 \\
9.7 \pm 3.3\end{array}$ & $5.7-19.2$ & $12.5 \pm 4.7$ & $5.2-24.0$ & $0.012 *$ & -1.182 \\
\hline Wilmore and Behnke [20] & $15.4 \pm 4.1$ & $9.3-24.3$ & $14.2 \pm 3.6$ & $9.5-21.8$ & $16.3 \pm 4.2$ & $9.3-24.3$ & $0.050^{*}$ & -0.537 \\
\hline Withers et al. [21] & $13.5 \pm 5.1$ & $6.5-26.2$ & $11.9 \pm 4.1$ & $7.0-24.4$ & $14.6 \pm 5.4$ & $6.5-26.2$ & 0.037 * & -0.563 \\
\hline Skeletal muscle mass (kg), Lee et al. [23] & $33.1 \pm 3.9$ & $25.4-44.1$ & $33.9 \pm 4.2$ & $26.2-44.1$ & $32.5 \pm 3.7$ & 25.4-41.4 & 0.181 & 0.354 \\
\hline Total upper-arm area $\left(\mathrm{cm}^{2}\right)$ & $79.6 \pm 12.5$ & $53.0-109.5$ & $81.1 \pm 13.1$ & $55.0-109.5$ & $78.5 \pm 12.1$ & $53.0-104.9$ & 0.422 & 0.206 \\
\hline Upper-arm muscle area $\left(\mathrm{cm}^{2}\right)$ & $67.4 \pm 10.7$ & $47.8-94.1$ & $69.9 \pm 11.4$ & $49.2-90.3$ & $65.6 \pm 10.0$ & $47.8-94.1$ & 0.132 & 0.401 \\
\hline Upper-arm fat area $\left(\mathrm{cm}^{2}\right)$ & $12.2 \pm 5.3$ & $5.2-31.6$ & $11.3 \pm 4.7$ & $5.9-26.7$ & $12.9 \pm 5.7$ & $5.2-31.6$ & 0.257 & -0.306 \\
\hline Total thigh area $\left(\mathrm{cm}^{2}\right)$ & $231.2 \pm 28.9$ & $169.9-302.9$ & $232.1 \pm 27.4$ & $177.3-302.9$ & $230.5 \pm 30.3$ & $169.9-290.3$ & 0.844 & 0.055 \\
\hline Thigh muscle area $\left(\mathrm{cm}^{2}\right)$ & $198.4 \pm 26.5$ & $142.8-274.6$ & $204.5 \pm 27.9$ & $154.0-274.6$ & $194.0 \pm 25.0$ & $142.8-247.3$ & 0.131 & 0.396 \\
\hline
\end{tabular}


Table 1. Cont.

\begin{tabular}{|c|c|c|c|c|c|c|c|c|}
\hline \multirow{2}{*}{ Variables } & \multicolumn{2}{|c|}{ Total Padel Players $(n=60)$} & \multicolumn{2}{|c|}{ Elite $(n=25)$} & \multicolumn{2}{|c|}{ Subelite $(n=35)$} & \multirow{2}{*}{$p$-Value } & \multirow{2}{*}{ Cohen's $d$} \\
\hline & Mean \pm SD & Range & Mean \pm SD & Range & Mean \pm SD & Range & & \\
\hline Thig fat area $\left(\mathrm{cm}^{2}\right)$ & $32.8 \pm 13.9$ & $14.8-82.7$ & $27.5 \pm 9.4$ & $14.8-55.9$ & $36.6 \pm 15.4$ & $15.5-82.7$ & $0.007^{*}$ & -0.713 \\
\hline Somatotype & & & & & & & & \\
\hline Endomorphy & $4.1 \pm 1.5$ & $1.8-7.9$ & $3.7 \pm 1.3$ & $2.0-7.3$ & $4.3 \pm 1.5$ & $1.8-7.9$ & 0.126 & -0.427 \\
\hline Mesomorphy & $5.4 \pm 1.1$ & $2.6-8.0$ & $5.7 \pm 1.2$ & $2.7-8.0$ & $5.3 \pm 0.9$ & $3.5-7.3$ & 0.138 & 0.377 \\
\hline Ectomorphy & $2.1 \pm 1.1$ & -0.6 to 5.4 & $2.0 \pm 1.1$ & -0.6 to 5.4 & $2.1 \pm 1.1$ & $0.0-4.3$ & 0.879 & -0.090 \\
\hline \multicolumn{9}{|l|}{ Hand grip strength } \\
\hline Dominant hand $(\mathrm{kg})$ & $49.4 \pm 9.7$ & $36.3-85.2$ & $51.3 \pm 12.5$ & $36.6-85.2$ & $48.1 \pm 7.1$ & $36.3-69.9$ & 0.268 & 0.315 \\
\hline Nondominant hand (kg) & $42.7 \pm 6.6$ & $25.7-63.8$ & $43.6 \pm 8.2$ & $25.7-63.8$ & $42.1 \pm 5.3$ & $31.0-52.7$ & 0.407 & 0.217 \\
\hline Sum two hands $(\mathrm{kg})$ & $92.1 \pm 14.8$ & $65.1-149.0$ & $94.9 \pm 18.8$ & $65.1-149.0$ & $90.2 \pm 11.2$ & $67.3-122.6$ & 0.239 & 0.304 \\
\hline Lumbar isometric strength $(\mathrm{kg})$ & $135.2 \pm 37.4$ & $28.0-275.0$ & $147.1 \pm 43.5$ & $68.0-275.0$ & $126.9 \pm 30.4$ & $28.0-186.0$ & $0.042 *$ & 0.538 \\
\hline Flexibility $(\mathrm{cm})$ & $22.1 \pm 8.6$ & $4.0-41.0$ & $23.9 \pm 9.6$ & $4.0-41.0$ & $20.8 \pm 7.6$ & $4.0-35.0$ & 0.175 & 0.358 \\
\hline \multicolumn{9}{|l|}{ Lower-body muscular strength } \\
\hline CMJ height $(\mathrm{cm})$ & $43.3 \pm 5.9$ & $31.0-59.0$ & $44.6 \pm 5.3$ & $34.0-54.0$ & $42.3 \pm 6.2$ & $31.0-59.0$ & 0.159 & 0.399 \\
\hline CMJ power (W) & $2212.5 \pm 352.8$ & $1500.0-3311.0$ & $2257.0 \pm 336.2$ & $1500.0-3126.1$ & $2179.1 \pm 366.4$ & 1541.3-3311.0 & 0.418 & 0.222 \\
\hline
\end{tabular}

${ }^{*} p<0.05 ;{ }^{* *} p<0.001 ;+$ Relaxed; +† Tensed. CMJ: Countermovement jump. BMI: Body mass index. 
Mean $( \pm \mathrm{SD})$ hand grip strength of elite padel players overall was $51.3 \pm 12.2 \mathrm{~kg}$ for the dominant hand and $43.6 \pm 8.8 \mathrm{~kg}$ for the nondominant hand. Lumbar isometric strength, flexibility, and countermovement jump height were $147.1 \pm 43.5 \mathrm{~kg}, 23.9 \pm 9.6 \mathrm{~cm}$, and $44.6 \pm 5.3 \mathrm{~cm}$, respectively. An anthropometric and physical profile is given in Table 2. Scores for 24 anthropometric dimensions and four performance tests are located on the chart, together with corresponding percentile values.

Table 2. Percentiles for the anthropometric and physical variables of elite padel players $(\mathrm{n}=25)$.

\begin{tabular}{|c|c|c|c|c|c|c|c|}
\hline \multirow{2}{*}{ Variable } & \multicolumn{7}{|c|}{ Percentiles } \\
\hline & 5 & 10 & 25 & 50 & 75 & 90 & 95 \\
\hline Height $(\mathrm{cm})$ & 164.4 & 166.9 & 174.3 & 177.6 & 182.8 & 187.1 & 194.5 \\
\hline Weight (kg) & 58.1 & 64.9 & 71.3 & 77.1 & 81.2 & 94.2 & 97.5 \\
\hline BMI $\left(\mathrm{kg} \cdot \mathrm{m}^{-2}\right)$ & 19.2 & 21.6 & 23.1 & 24.3 & 25.6 & 27.5 & 30.3 \\
\hline Arm span $(\mathrm{cm})$ & 165.4 & 175.6 & 178.8 & 181.8 & 184.7 & 192.8 & 201.0 \\
\hline Triceps skinfold (mm) & 5.6 & 6.1 & 7.4 & 8.4 & 13.2 & 17.1 & 21.0 \\
\hline Biceps skinfold (mm) & 2.4 & 2.5 & 2.9 & 4.0 & 4.9 & 6.7 & 7.8 \\
\hline Subscapular skinfold (mm) & 6.5 & 7.0 & 7.6 & 9.4 & 11.2 & 13.0 & 22.9 \\
\hline Suprailiac skinfold (mm) & 9.6 & 10.3 & 12.6 & 16.6 & 22.4 & 30.3 & 33.4 \\
\hline Supraespinale skinfold (mm) & 4.3 & 4.7 & 5.6 & 7.4 & 11.1 & 14.6 & 20.2 \\
\hline Abdominal skinfold (mm) & 6.4 & 7.1 & 9.7 & 13.2 & 23.4 & 30.7 & 35.1 \\
\hline Thigh skinfold $(\mathrm{mm})$ & 5.9 & 6.2 & 8.2 & 9.6 & 12.4 & 15.6 & 20.9 \\
\hline Calf skinfold (mm) & 4.2 & 4.3 & 5.0 & 5.8 & 8.6 & 10.3 & 11.7 \\
\hline Upper-arm girth $(\mathrm{cm}) \dagger$ & 26.8 & 28.2 & 30.1 & 31.5 & 33.6 & 35.2 & 36.6 \\
\hline Upper-arm girth $(\mathrm{cm})++$ & 28.6 & 29.9 & 31.9 & 34.2 & 35.4 & 37.7 & 38.0 \\
\hline Right forearm girth $(\mathrm{cm})$ & 25.9 & 26.2 & 27.1 & 28.6 & 30.3 & 32.3 & 33.1 \\
\hline Left forearm girth $(\mathrm{cm})$ & 23.7 & 24.9 & 26.6 & 27.5 & 28.7 & 30.3 & 31.0 \\
\hline Wrist girth $(\mathrm{cm})$ & 15.7 & 16.1 & 16.7 & 17.6 & 18.3 & 19.2 & 19.9 \\
\hline Thigh girth $(\mathrm{cm})$ & 47.9 & 50.2 & 51.3 & 53.7 & 56.0 & 58.0 & 60.8 \\
\hline Calf girth (maximum) $(\mathrm{cm})$ & 33.1 & 34.7 & 36.4 & 38.1 & 39.5 & 41.2 & 41.6 \\
\hline Humerus breath $(\mathrm{cm})$ & 6.3 & 6.6 & 6.9 & 7.4 & 7.6 & 8.1 & 8.3 \\
\hline Femur breath $(\mathrm{cm})$ & 9.3 & 9.4 & 9.7 & 10.1 & 10.5 & 10.8 & 11.2 \\
\hline Dominant hand length $(\mathrm{cm})$ & 17.8 & 18.5 & 19.4 & 20.0 & 20.6 & 21.8 & 22.1 \\
\hline Dominant hand width $(\mathrm{cm})$ & 19.5 & 19.9 & 20.6 & 22.5 & 23.8 & 25.0 & 25.8 \\
\hline Nondominant hand length $(\mathrm{cm})$ & 16.8 & 18.0 & 19.3 & 19.7 & 20.4 & 21.4 & 22.5 \\
\hline Nondominant hand width $(\mathrm{cm})$ & 19.7 & 19.9 & 20.9 & 22.5 & 24.7 & 25.0 & 25.7 \\
\hline \multicolumn{8}{|l|}{ Hand grip strength } \\
\hline Dominant hand $(\mathrm{kg})$ & 37.3 & 39.5 & 42.9 & 46.5 & 57.8 & 74.1 & 84.1 \\
\hline Nondominant hand $(\mathrm{kg})$ & 27.7 & 33.6 & 38.7 & 43.1 & 47.5 & 55.9 & 62.7 \\
\hline Sum of two hands $(\mathrm{kg})$ & 66.4 & 73.2 & 83.1 & 91.3 & 102.9 & 126.2 & 143.5 \\
\hline Lumbar isometric strength (kg) & 75.1 & 101.8 & 120.8 & 143.5 & 160.1 & 216.8 & 263.5 \\
\hline Flexibility $(\mathrm{cm})$ & 4.5 & 9.5 & 19.0 & 24.0 & 30.8 & 39.0 & 40.8 \\
\hline \multicolumn{8}{|l|}{ Lower-body muscular strength } \\
\hline CMJ Height (cm) & 34.3 & 36.5 & 40.8 & 45.0 & 47.8 & 52.0 & 54.0 \\
\hline CMJ Power (W) & 1606.5 & 1959.1 & 2032.6 & 2213.9 & 2405.6 & 2788.1 & 3079.0 \\
\hline
\end{tabular}

† Relaxed; +† Flexed and tensed; CMJ: Countermovement jump.

\section{Discussion}

The association between anthropometric characteristics and physical fitness in sports performance has been widely described in scientific literature [26-28]. However, this is the first study to our knowledge that has examined differences between male padel players according to their competitive level.

Comparisons between elite and subelite groups revealed elite players to be older ( $31 \pm 5.7 \mathrm{vs}$. $25.3 \pm 5.9$ years $)$ with a significantly greater number of training years ( $15.0 \pm 6.1$ vs. $8.6 \pm 3.9$ years $)$. Furthermore, elite padel players showed lower values for thigh and calf skinfolds (10.6 $\pm 3.8 \mathrm{vs}$. $14.1 \pm 5.7 ; 6.7 \pm 2.3$ vs. $9.3 \pm 4.4$, respectively), the sum of six and eight skinfolds ( $84.5 \pm 30.7$ vs. $102.7 \pm 38.5 \mathrm{~mm} ; 95.3 \pm 33.3$ vs. $116 \pm 43.2 \mathrm{~mm}$, respectively), and the sum of lower-limb skinfolds $(17.3 \pm 5.4$ vs. $23.5 \pm 9.9 \mathrm{~mm})$, than the subelite padel players group. In addition, elite players showed lower $\mathrm{BF} \%$ (between 2.1 and 2.7\%) using three different equations [9-11], in addition to a lower thigh fat area $\left(-9.1 \mathrm{~cm}^{2}\right)$. This lower $\mathrm{BF} \%$ could be related to the higher amount of training undertaken by the elite group. No statistically significant differences were observed between groups for height, body weight, and BMI. The mean somatotype for elite padel players (3.7-5.7-2.0) demonstrates that these athletes were predominantly mesomorphic, being characterised as endo-mesomorphic according to Carter and Heath [24].

Table 3 shows the anthropometric characteristics of different racket spots according to performance level and age. 
Table 3. Summary table of studies examining age, stature, body weight, body mass index (BMI), body fat percentage and somatotype of elite players of different racket sports (mean $\pm \mathrm{SD}$ ).

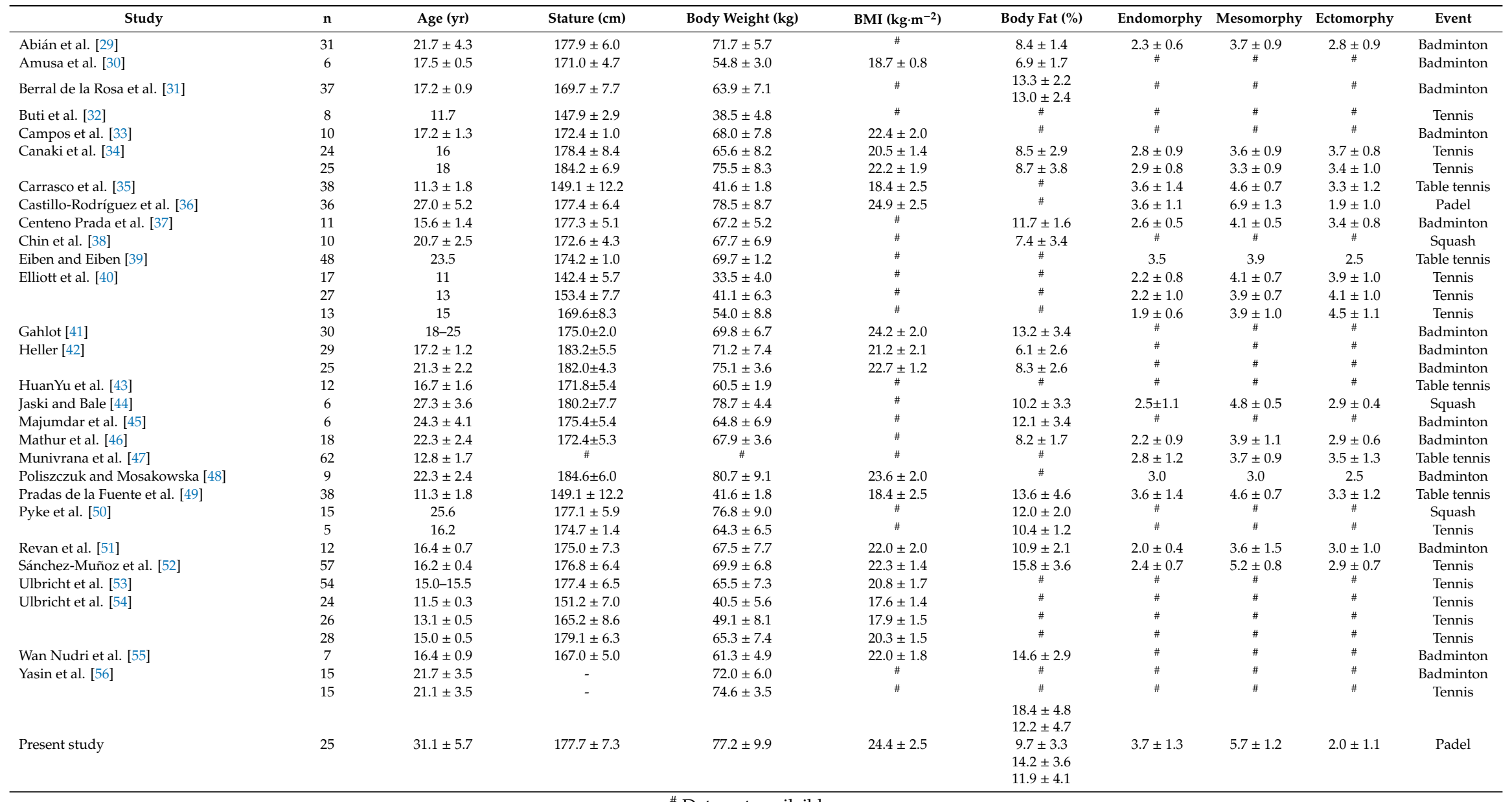

\# Data not availaible. 
With respect to padel players participating in a national university padel championship [5], players in our study were older $(31.1 \pm 5.7$ years vs. $23.1 \pm 3.6$ years), heavier $(77.2 \pm 9.9 \mathrm{~kg}$ vs. $74.3 \pm 8.6 \mathrm{~kg})$, and shorter $(177.7 \pm 7.3 \mathrm{~cm}$ vs. $180.0 \pm 10.0 \mathrm{~cm})$. We also found lower values for the thickness of all skinfolds, higher thigh and calf girths $(53.9 \pm 3.2 \mathrm{~cm}$ vs. $52.2 \pm 3.6 \mathrm{~cm}, 37.8 \pm 2.3 \mathrm{~kg}$ vs. $37.1 \pm 1.9 \mathrm{~cm}$, respectively), higher mesomorphic component (5.7 \pm 1.2 vs. $4.1 \pm 0.9)$, and similar endomorphic and mesomorphic components ( $3.7 \pm 1.3$ vs. $3.7 \pm 1.2,2.0 \pm 1.1$ vs. $2.4 \pm 0.8$, respectively). Similar results were observed by Castillo-Rodríguez et al. [36] in body weight, stature, and all components of somatotype. The only exception to this was found for the mesomorphic component, with this showing a lower value $(5.7 \pm 1.2$ vs. $6.9 \pm 1.3)$ than that reported for padel players in the present study.

We detected important differences between current elite padel players and athletes from other racket sports. Elite padel players were heavier $(77.2 \pm 9.9 \mathrm{~kg}$ vs. $71.7 \pm 5.7 \mathrm{~kg})$, with similar stature, higher skinfold thickness, and lower thigh girth than that observed in Spanish badminton players [29]. In addition, they showed higher endomorphic and mesomorphic components, and a lower ectomorphic component $(3.7 \pm 1.3$ vs. $2.3 \pm 0.6,5.7 \pm 1.2$ vs. $3.7 \pm 0.9,2.0 \pm 1.1$ vs. $2.8 \pm 0.9$, respectively) than badminton players. Conversely, in comparison with previous reports in squash [44], current elite padel players were shorter $(177.7 \pm 7.3 \mathrm{~cm}$ vs. $180.0 \pm 10.0 \mathrm{~cm})$ and lighter $(77.2 \pm 9.9 \mathrm{~kg}$ vs. $78.7 \pm 4.4 \mathrm{~kg}$ ), showed higher values for skinfold thickness (biceps: $4.1 \pm 1.5 \mathrm{~mm}$ vs. $3.8 \pm 1.4 \mathrm{~mm}$; triceps: $10.5 \pm 4.4 \mathrm{~mm}$ vs. $6.7 \pm 3.0 \mathrm{~mm}$; subscapular: $10.1 \pm 4.1 \mathrm{~mm}$ vs. $8.6 \pm 2.1 \mathrm{~mm}$; suprailiac: $18.3 \pm 7.2 \mathrm{~mm}$ vs. $5.7 \pm 2.2 \mathrm{~mm}$; thigh: $10.6 \pm 3.8 \mathrm{~mm}$ vs. $9.1 \pm 4.3 \mathrm{~mm}$; and calf: $6.7 \pm 2.3 \mathrm{~mm}$ vs. $4.0 \pm 2.5 \mathrm{~mm})$, and a lower thigh girth $(53.9 \pm 3.2 \mathrm{~cm}$ vs. $58.5 \pm 0.5 \mathrm{~cm})$. Furthermore, mean somatotype of padel players in the present study was highly endomorphic and mesomorphic, but less ectomorphic $(3.7 \pm 1.3$ vs. $2.5 \pm 1.1,5.7 \pm 1.2$ vs. $4.8 \pm 0.5,2.0 \pm 1.1$ vs. $2.9 \pm 0.4$, respectively). In comparison to table tennis players [29], elite padel players were taller and heavier $(177.7 \pm 7.3 \mathrm{~cm}$ vs. $174.2 \pm 1.0 \mathrm{~cm}, 77.2 \pm 9.9 \mathrm{~kg}$ vs. $69.7 \pm 1.2 \mathrm{~kg}$, respectively), and showed a different somatotype with a greater endomorphic component ( $5.7 \pm 1.2 \mathrm{vs}$. 3.9). Similarly, in comparison to subelite tennis players [6], the current padel players were taller and heavier $(177.7 \pm 7.3 \mathrm{~cm}$ vs. $180.0 \pm 10.0 \mathrm{~cm}$, $77.2 \pm 9.9 \mathrm{~kg}$ vs. $74.7 \pm 7.5 \mathrm{~kg}$, respectively), showing similar somatotype components. The only exception in this case was seen in the mesomorphic component, with padel players showing higher values ( $5.7 \pm 1.2$ vs. $4.3 \pm 1.2$ ). Competition level may, however, account for these differences. Secondand third-division tennis players [57] were taller (184.8 $\pm 4.8 \mathrm{~cm}$ vs. $177.7 \pm 7.3 \mathrm{~cm})$, showed lower values for skinfold thickness (triceps: $7.3 \pm 2.8 \mathrm{~mm}$ vs. $10.5 \pm 4.4 \mathrm{~mm}$; subscapular: $9.7 \pm 1.9 \mathrm{~mm}$ vs. $10.1 \pm 4.1 \mathrm{~mm}$; suprailiac: $8.4 \pm 2.7 \mathrm{~mm}$ vs. $18.3 \pm 7.2 \mathrm{~mm}$; thigh: $6.8 \pm 2.0 \mathrm{~mm}$ vs. $10.6 \pm 3.8 \mathrm{~mm}$; and calf: $7.8 \pm 2.7 \mathrm{~mm}$ vs. $6.7 \pm 2.3 \mathrm{~mm})$, and a lower thigh girth $(52.4 \pm 3.0 \mathrm{~cm}$ vs. $53.9 \pm 3.2 \mathrm{~cm})$ than elite padel players. Nevertheless, elite padel players showed higher endomorphic and mesomorphic components and a lower ectomorphic component ( $3.7 \pm 1.3$ vs. $2.3 \pm 0.6,5.7 \pm 1.2$ vs. $3.2 \pm 0.9,2.0 \pm 1.1$ vs. $3.1 \pm 0.8$, respectively) than nonelite tennis players [11].

A player's height and arm span is highly important in padel. High power is required to successfully bring rallies to a close, with the most powerful plays being found when players hit the ball when it is high above the head, as is often seen in tennis [5]. In this sense, it would be useful for padel players to be tall and of relatively high weight (higher muscle weight, of course) so that they are able to perform very powerful hits, especially when they start a point or when they want to finish it suddenly with a powerful shot (which can sometimes take the ball out of court). This situation does not normally happen in squash or in table-tennis. Padel players typically need to move their position more than squash or table tennis players, covering a bigger space. In addition, whilst they do not normally need such a fast reaction time, they do require high power in their legs to quickly shift their upper-body weight.

A main contribution of the current study was the measurement of physical fitness in world-class padel players. In contrast to what was observed in other racket sports like tennis or squash [9], no significant differences were found between elite and subelite groups in analysed fitness variables (CMJ, handgrip, and flexibility). The only exception was seen with lumbar isometric strength, which was 
greater in elite padel players $(147.1 \pm 43.5 \mathrm{~kg}$ vs. $126.9 \pm 30.4 \mathrm{~kg})$. Similar physical fitness between highand low-level players has been previously observed in nonprofessional padel samples $[12,13]$. This appears to indicate that technical, tactical, and psychological aspects should become topics of interest in padel performance training and assessment. For instance, smashing abilities from middle distances (a low-frequency action $<10 \%$ of the game, but executed at high speed) and volleying effectively close to the net (a high-frequency action $>30 \%$ of the game and made in a short time frame) appear to be relevant for increasing point scoring [5]. In this sense, a stronger core musculature seems to be important in padel to allow players to perform powerful strokes. According to our results, better lumbar isometric strength could help padel players avoid lower back pain and develop greater hitting force when playing overhead groundstrokes involving ballistic trunk movements, such as a smash. This has also been reported in tennis [58].

\section{Limitations}

Results must be considered as a reference point, as opposed to being used as an obligatory model of better performance. In this way, the results presented can be used as a standard reference, but should be interpreted with caution according to individual characteristics and needs.

Strengths: The main strengths of the present study are that a) it is the first time that anthropometrical, somatotype, and body composition variables were measured in top-level padel players; $b$ ) participants were involved in performing padel at a high level (they were the best padel players in the world at the time of study); c) the best padel players were compared with lower ranked players in order to ascertain whether differences could be found with regards to anthropometric variables; d) the study was carried out during one of the most important competitions in the world for elite padel players and so all participants should have been in top physical shape.

Future lines: a) To conduct a longitudinal study to investigate anthropometry, body composition, and somatotype of padel players at different competitive levels; b) to investigate nutritional habits and profiles in elite padel players, and their relationship with body composition and sports training.

Practical applications: This study provides reference values in relation to the anthropometric and physical characteristics of elite padel players. This information provides a frame of reference for coaches to control the training process in order to improve athletes' performance and to facilitate talent detection and identification in padel.

\section{Conclusions}

In summary, the present study examined differences in the anthropometric characteristics and physical performance of padel players according to their competitive level (elite and subelite). Elite players showed lower values than subelite padel players for thigh and calf skinfolds, the sum of 6 and 8 skinfolds, and the sum of lower-limb skinfolds. Elite players also showed lower BF\%. This study provides normative data that could help coaches with talent detection. Result may also help coaches in the design of training programs in order to achieve maximum sports performance.

Author Contributions: Conceptualization, C.S.-M. and M.Z.; Investigation, C.S.-M., J.C., J.C.-I., B.J.S.-A., and M.Z.; Methodology, C.S.-M. and J.J.M.; Supervision, M.Z.; Writing-original draft, C.S.-M. and J.J.M.; Writing一review and editing, J.C., J.C.-I., B.J.S.-A., and M.Z. All authors have read and agree to the published version of the manuscript.

Funding: This research received no external funding.

Acknowledgments: The authors would like to thank Padel Pro Tour for their permission to set up this study and for their full support and collaboration during the investigation. The authors also wish to thank all padel players for their participation and cooperation in this study, and Jaime Morente for his technical support.

Conflicts of Interest: The authors have no conflicts of interest. 


\section{References}

1. Courel-Ibáñez, J.; Sánchez-Alcaraz, B.J.; García Benítez, S.; Echegaray, M. Evolution of padel in Spain according to practitioners' gender and age. CCD 2017, 12, 39-46. [CrossRef]

2. Courel-Ibáñez, J.; Sánchez-Alcaraz, B.J.; Cañas, J. Game performance and length of rally in professional padel player. J. Hum. Kinet. 2017, 55, 161-169. [CrossRef]

3. Carrasco, L.; Romero, S.; Sañudo, B.; De Hoyo, M. Game analysis and energy requirements of paddle tennis competition. Sci. Sports 2011, 26, 338-344. [CrossRef]

4. Castillo-Rodríguez, A.; Alvero-Cruz, J.R.; Hernández-Mendo, A.; Fernández-García, J.C. Physical and physiological responses in Paddle Tennis competition. Int. J. Perform. Anal. Sport 2014, 14, 524-534. [CrossRef]

5. Courel-Ibáñez, J.; Sánchez-Alcaraz Martinez, B.J.; Muñoz Marín, D. Exploring game dynamics in padel. Implications for assessment and training. J. Strength Cond. Res. 2019, 33, 1971-1977. [CrossRef]

6. García-Benítez, S.; Courel-Ibáñez, J.; Pérez-Bilbao, T.; Felipe, J.L. Game responses during young padel match play: Age and sex comparisons. J. Strength Cond. Res. 2018, 32, 1144-1149. [CrossRef]

7. Torres-Luque, G.; Ramirez, A.; Cabello-Manrique, D.; Nikolaidis, P.T.; Alvero-Cruz, J.R. Match analysis of elite players during paddle tennis competition. Int. J. Perform. Anal. Sport 2015, 15, 1135-1144. [CrossRef]

8. Fernandez-Fernandez, J.; Sanz-Rivas, D.; Mendez-Villanueva, A. A review of the activity profile and physiological demands of tennis match play. Strength Cond. J. 2009, 31, 15-26. [CrossRef]

9. Jones, T.W.; Williams, B.K.; Kilgallen, C.; Horobeanu, C.; Shillabeer, B.C.; Murray, A.; Cardinale, M. A review of the performance requirements of squash. Int. J. Sports Sci. Coach. 2018, 13, 1223-1232. [CrossRef]

10. Söğüt, M. Stature: Does it really make a difference in match-play outcomes among professional tennis players? Int. J. Perform. Anal. Sport 2018, 18, 255-261. [CrossRef]

11. Wong, F.K.H.; Keung, J.H.; Lau, N.M.; Ng, D.K.; Chung, J.W.; Chow, D.H. Effects of body mass index and full body kinematics on tennis serve speed. J. Hum. Kinet. 2014, 40, 21-28. [CrossRef]

12. Courel-Ibáñez, J.; Herrera-Gálvez, J.J. Fitness testing in padel: Performance differences according to players' competitive level. Sci. Sports 2019. [CrossRef]

13. Müller, C.B.; Vecchio, F.B. Del Physical fitness of amateur paddle tennis players: Comparisons between different competitive levels. Motricidade 2018, 14, 42-51. [CrossRef]

14. Gale-Watts, A.S.; Nevill, A.M. From endurance to power athletes: The changing shape of successful male professional tennis players. Eur. J. Sport Sci. 2016, 16, 948-954. [CrossRef]

15. Martínez-Rodriguez, A.; Roche Collado, E.; Vicente-Salar, N. Body composition assessment of paddle and tennis adult male players. Nutr. Hosp. 2015, 31, 1294-1301.

16. Marfell-Jones, M.; Olds, T.; Stewart, A.; Carter, L. International Standards for Anthropometric Assessment; ISAK: Potchefstroom, South Africa, 2006.

17. Durnin, J.V.; Womersley, J. Body fat assessed from total body density and its estimation from skinfold thicknesses measurements on 481 and women aged 16-72 years. Br. J. Nutr. 1974, 32, 77-97. [CrossRef]

18. Katch, F.I.; McArdle, W.D. Prediction of body density from simple anthropometric measurements in college-age men and women. Hum. Biol. 1973, 45, 445-455.

19. Sloan, A.W. Estimation of body fat in young men. J. Appl. Physiol. 1967, 23, 311-315. [CrossRef]

20. Wilmore, J.H.; Behnke, A.R. An anthropometric estimation of body density and lean body weight in young men. J. Appl. Physiol. 1969, 27, 25-31. [CrossRef]

21. Withers, R.T.; Craig, N.P.; Bourdon, P.C.; Norton, K.I. Relative body fat and anthropometric prediction of body density of male athletes. Eur. J. Appl. Physiol. 1987, 56, 191-200. [CrossRef]

22. Siri, W.E. Body composition from fluid space and density. In Techniques for Measuring Body Composition; Brozek, J., Hanschel, A., Eds.; National Academy of Science: Washington DC, USA, 1961; pp. 223-224.

23. Lee, R.C.; Wang, Z.; Heo, M.; Ross, R.; Janssen, I.; Heymsfield, S.B. Total-body skeletal muscle mass: Development and cross-validation of anthropometric prediction models. Am. J. Clin. Nutr. 2000, 72, 796-803. [CrossRef] [PubMed]

24. Carter, J.E.L.; Heath, B.H. Somatotyping: Development and Applications; Cambridge University Press: Cambridge, UK, 1990.

25. González-Badillo, J.J.; Gorostiaga-Ayestarán, E. Foundations of Strength Training. In Application to Sport Performance; Inde: Barcelona, Spain, 1997. 
26. Mostaert, M.; Deconinck, F.; Pion, J.; Lenoir, M. Anthropometry, physical fitness and coordination of young figure skaters of different levels. Int. J. Sports Med. 2016, 37, 531-538. [CrossRef] [PubMed]

27. Sarah, G.L.; Kenji, D.; Wade, S.; Harry, B.G.; Carl, W.T. Identifying the physical fitness, anthropometric and athletic movement qualities discriminant of developmental level in elite junior Australian football: Implications for the development of talent. J. Strength Cond. Res. 2016, 31, 1830-1839.

28. Schwesig, R.; Hermassi, S.; Fieseler, G.; Irlenbusch, L.; Noack, F.; Delank, K.S.; Shephard, R.J.; Chelly, M.S. Anthropometric and physical performance characteristics of professional handball players: Influence of playing position and competitive level. J. Sports Med. Phys. Fit. 2016, 57, 1471-1478.

29. Abián, V.P.; Abián-Vicén, J.; Sampedro-Molinuevo, J. Anthropometric analysis of body symmetry in badminton players. Int. J. Morphol. 2012, 30, 945-951. [CrossRef]

30. Amusa, L.O.; Toriola, A.L.; Dhaliwal, H.S.; Mokgwathi, M.M. Anthropometric profile of Botswana junior national badminton players. J. Hum. Mov. Stud. 2001, 40, 115-128.

31. Berral de la Rosa, F.J.; Rodríguez-Bies, E.C.; Berral de la Rosa, C.J.; Rojano, D.; Lara, E. Comparison of anthropometric equations for estimation muscle mass in badminton player. Int. J. Morphol. 2010, 28, 803-810.

32. Buti, T.; Elliott, B.; Morton, A. Physiological and anthropometric profiles of elite prepubescent tennis players. Physician Sportsmed. 1984, 12, 111-116. [CrossRef]

33. Campos, F.A.D.; Daros, L.B.; Mastrascusa, V.; Dourado, A.C.; Stanganelli, L.C.R. Anthropometric profile and motor performance of junior badminton players. Braz. J. Biomotricity 2009, 3, 146-151.

34. Canaki, M.; Sporis, G.; Leko, G. Morphological advantages and disadvantages in Croatian U-16 and U-18 tennis players. Hrvat. Sportskomed. Vjesn. 2006, 21, 98-102.

35. Carrasco, L.; Pradas, F.; Martínez, A. Somatotype and body composition of young top-level table tennis players. Int. J. Table Tennis. Sci. 2010, 6, 175-177.

36. Castillo-Rodríguez, A.; Hernández-Mendo, A.; Alvero-Cruz, J.R. Morphology of the elite paddle player-Comparison with other racket sports. Int. J. Morphol. 2014, 32, 177-182. [CrossRef]

37. Centeno Prada, R.A.; Naranjo Orellana, J.; Guerra Moreno, V. Kinanthropometric study of elite badminton young players. AMD 1999, 70, 115-119.

38. Chin, M.K.; Steininger, K.; So, R.C.H.; Clark, C.R.; Wong, A.S.K. Physiological profiles and sport specific fitness of Asian elite squash players. Br. J. Sports Med. 1995, 29, 158-164. [CrossRef]

39. Eiben, O.G.; Eiben, E. The physique of European table-tennis players. Coll. Antropol. 1979, 1, 67-76.

40. Elliott, B.C.; Ackland, T.R.; Blanksby, B.A.; Bloomfield, J. A prospective study of physiological and kinanthropometric indicators of junior tennis performance. Aust. J. Sci. Med. Sport 1990, 22, 87-92.

41. Gahlot, P. A study on physiological characteristics of elite and sub-elite male badminton players of Delhi region. Int. Res. J. Manag. Sociol. Hum. 2016, 7, 209-214.

42. Heller, J. Physiological profiles of elite badminton players aspects of age and gender. Br. J. Sports Med. 2010, 44, 1S-13S. [CrossRef]

43. HuanYu, Z.; Ushiyama, Y.; Fei, Y.; Iizuka, S.; Kamijima, K. Estimation of energy consumption from heart rates of Chinese professional table tennis players in training conditions. Int. J. Table Tennis Sci. 2010, 6, 139-144.

44. Jaski, A.; Bale, P. The physique and body composition of top class squash players. J. Sports Med. 1987, 27, 114-118.

45. Majumdar, P.; Khanna, G.L.; Malik, V.; Sachdeva, S.; Arif, M.; Mandal, M. Physiological analysis to quantify training load in badminton. Br. J. Sports Med. 1997, 31, 342-345. [CrossRef] [PubMed]

46. Mathur, D.N.; Toriola, A.L.; Igbokwe, N.U. Somatotypes of Nigerian athletes of several sports. Br. J. Sports Med. 1985, 19, 219-220. [CrossRef] [PubMed]

47. Munivrana, G.; Pausic, J.; Kondric, M. The influence of somatotype on young table tennis players' competitive success. Kinesiol. Sloven. 2011, 17, 42-51.

48. Poliszczuk, T.; Mosakowska, M. Antropometryczny profil elitarnych badmintonisto w z Polski. Med. Sport 2010, 1, 45-55.

49. Pradas de la Fuente, F.; Carrasco-Páez, L.; Martínez-Pardo, E.; Herrero-Pagán, R. Anthropometric profile, somatotype, and body composition of young table tennis players. Int. J. Sport Sci. 2007, 7, 11-23.

50. Pyke, S.; Elliott, C.; Pyke, E. Performance testing of tennis and squash players. Br. J. Sports Med. 1974, 8, 80-86. [CrossRef] 
51. Revan, S.; Aydogmus, M.; Balci, S.S.; Pepe, H.; Eroglu, H. The evaluation of some physical and physiological characteristics of Turkish and foreign national badminton team players. J. Phys. Educ. Sport Sci. 2007, 1, 63-70.

52. Sánchez-Muñoz, C.; Sanz, D.; Zabala, M. Anthropometric characteristics, body composition and somatotype of elite junior tennis players. Br. J. Sports Med. 2007, 41, 793-799. [CrossRef]

53. Ulbricht, A.; Fernandez-Fernandez, J.; Mendez-Villanueva, A.; Ferrauti, A. Impact of fitness characteristics on tennis performance in elite junior tennis players. J. Strength Cond. Res. 2016, 30, 989-998. [CrossRef]

54. Ulbricht, A.; Fernandez-Fernandez, J.; Ferrauti, A. Conception for fitness testing and individualized training programs in the German Tennis Federation. Sports Orthop. Traum. 2013, 29, 180-192. [CrossRef]

55. Wan Nudri, W.D.; Ismail, M.N.; Zawiak, H. Anthropometric measurements and body composition of selected national athletes. Malays. J. Nutr. 1996, 2, 138-147. [PubMed]

56. Yasin, A.; Omer, S.; Ibrahim, Y.; Akif, B.M.; Cengiz, A. Comparison of some anthropometric characteristics of elite badminton and tennis players. Sci. Mov. Health 2010, 2, 400S-405S.

57. Raschka, C.; Schmidt, K. Sports anthropological and somatotypical comparison between higher class male and female badminton and tennis players. Pap. Anthropol. 2013, 22, 153-161. [CrossRef]

58. Campbell, A.; Straker, L.; O'Sullivan, P.; Elliot, B.; Reid, M. Lumbar loading in the elite adolescent tennis serve: Link to low back pain. Med. Sci. Sports Exerc. 2013, 45, 1562-1568. [CrossRef]

(C) 2020 by the authors. Licensee MDPI, Basel, Switzerland. This article is an open access article distributed under the terms and conditions of the Creative Commons Attribution (CC BY) license (http://creativecommons.org/licenses/by/4.0/). 